\title{
Equations of quantum theory in the space of randomly joint quantum events
}

\author{
Alexander Biryukov ${ }^{1, *}$ \\ ${ }^{1}$ Samara University
}

\begin{abstract}
The dynamics of the system in the space of random joint events is considered. The symmetric difference of events is introduced in space based on the Kolmogorov axioms. To describe quantum effects in the dynamics of the system, an additional axiom is introduced for random joint events: "the symmetric sum of random events." In the generated space of random joint events, an equation is constructed for the probability of a system transition between two events. It is shown that for pairwise joint events it is equivalent to the equation of quantum mechanics.
\end{abstract}

\section{Introduction}

The development of modern information and computer technologies based on quantum theory stimulate its further study from the point of view of probability theory, for example, works [1] - [7], which present original results and reviews in this direction. We propose to consider stochastic processes in the space of joint random events. The equations for the transition probabilities of systems in these spaces were proposed in [8] - [11]. We propose in this paper a model of the space of joint random events of classical and quantum physics, and equations for the probabilities of transitions between events. It is shown that the proposed equations for the probabilities of stochastic processes in the space of joint events, in the case when the events are only pairwise compatible, describe the dynamics of quantum processes.

\section{Joint random events in classical and quantum physics}

Consider the space $N$ of random joint events: $S_{n}, n=1,2, \ldots, N$. A model of the space in which all random events $S_{n}, n=1,2, \ldots, N$. are joint, is determined by the fact that there are intersections of these events, the probabilities of which are nonzero:

$$
P\left(S_{n} \cap S_{m}\right) \neq 0, \quad P\left(S_{n} \cap S_{m} \cap S_{k}\right) \neq 0, \ldots, P\left(S_{1} \cap S_{2} \cap \ldots \cap S_{N}\right) \neq 0,
$$

all indices $k, n, m, \ldots$ take values from 0 to $N$.

In the space of random joint events $S_{1}, S_{2}, \ldots, S_{N}$, we construct the symmetric difference of the events $S_{1}^{-}, S_{2}^{-}, \ldots, S_{N}^{-}$. The event $S_{n}^{-}$means that only the event with the corresponding number $n$ will occur, the event with a different number will not happen. The events

\footnotetext{
*e-mail: biryukov_1@mail.ru
} 
$S_{1}^{-}, S_{2}^{-}, \ldots, S_{N}^{-}$are not joint. The probability of their combination $P\left(S_{1}^{-} \cup S_{2}^{-} \cup \ldots \cup S_{N}^{-}\right)$is expressed in terms of probabilities of the events $S_{n}$, and probabilities of their intersection

$$
\begin{gathered}
P\left(\bigcup_{n=1}^{N} S_{n}^{-}\right)=\sum_{n=1}^{N} P\left(S_{n}\right)-2 \sum_{n<m=1}^{N} P\left(S_{n} \cap S_{m}\right)+ \\
+4 \sum_{n<m<k=1}^{N} P\left(S_{n} \cap S_{m} \cap S_{k}\right)+\ldots+2^{N-1}(-1)^{N-1} P\left(S_{1} \cap S_{2} \cap \ldots \cap S_{N}\right) .
\end{gathered}
$$

Expression (2) is proved in the axiomatics of Kolmogorov probability theory [12] - [15].

An analysis of the experiments shows that in the physics of the microworld there are joint random events that are described in the framework of the Kolmogorov axiomatics. However, from the analysis of experiments, for example, on electron diffraction, it follows that, there are joint random events, the description of which requires the introduction of an additional postulate to Kolmogorov's axioms. An additional postulate boils down to the assertion that there exists a symmetric sum of events $S_{1}^{+}, S_{2}^{+}, \ldots, S_{N}^{+}$. Their properties differ from those of Kolmogorov random joint events. The probability of their combination $P\left(S_{1}^{+} \cup S_{2}^{+} \cup \ldots \cup S_{N}^{+}\right)$ is expressed in terms of the probabilities of events $S_{n}, n=1,2, \ldots, N$, and their intersections (1) by the equation

$$
\begin{gathered}
P\left(\bigcup_{n=1}^{N} S_{n}^{+}\right)=\sum_{n=1}^{N} P\left(S_{n}\right)+2 \sum_{n<m=1}^{N} P\left(S_{n} \cap S_{m}\right)- \\
-4 \sum_{n<m<k=1}^{N} P\left(S_{n} \cap S_{m} \cap S_{k}\right)+\ldots+2^{N-1}(-1)^{N} P\left(S_{1} \cap S_{2} \cap \ldots \cap S_{N}\right) .
\end{gathered}
$$

Note, that the interpretation of events in equation (3) by sets, as provided by the Kolmogorov axiomatics, is unacceptable, since it leads to a contradiction.

Equations (2), (3) can be written in the form of a single equation. To this end we introduce the events $\tilde{S}_{n}$ each of which takes the value either $S_{n}^{-}$or $S_{n}^{+}$. The probability of combining events $\tilde{S}_{n}$ represented by the formula, which is the union of equations (2), (3):

$$
\begin{gathered}
P\left(\bigcup_{n=1}^{N} \tilde{S}_{n}\right)=\sum_{n=1}^{N} P\left(S_{n}\right)+2 \sum_{n<m=1}^{N} g_{n m} P\left(S_{n} \cap S_{m}\right)+ \\
+4 \sum_{n<m<k=1}^{N} g_{n m k} P\left(S_{n} \cap S_{m} \cap S_{k}\right)+\ldots+2^{N-1} g_{12 \ldots N} P\left(S_{1} \cap S_{2} \cap \ldots \cap S_{N}\right),
\end{gathered}
$$

where the factors $g_{n m}, g_{n k m}$ and others take one of the values $+1,-1$ depending on selected events $S_{n}, S_{k}, S_{m}, \ldots$.

\section{The equation for the probability of system transitions in the space of joint events}

We construct a model of the stochastic process of the system in a finite-dimensional space of random joint events.

Suppose that at the initial moment of time $t=0$ the system was in a certain state $A_{\text {in }}$, that is, the probability of the state $P\left(A_{\text {in }}=1\right)$. At time $t>0$ it is in the state $B_{f}$. In the period from the initial instant of time $t=0$ to the instant of time $t>0$, the state of the system is 
characterized by one of the events $\tilde{S}_{n}, n=1,2, \ldots, N$. The relationship between events is represented by the equation

$$
B_{f} \cap A_{\text {in }}=B_{f} \cap\left(\bigcup_{n=1}^{N} \tilde{S}_{n}\right) \cap A_{\text {in }}=\bigcup_{n=1}^{N}\left(B_{f} \cap \tilde{S}_{n} \cap A_{\text {in }}\right)=\bigcup_{n=1}^{N} \tilde{S}_{f n i} .
$$

We will designate $B_{f} \cap \tilde{S}_{n} \cap A_{\text {in }}=\tilde{S}_{f n i}, B_{f} \cap S_{n} \cap A_{\text {in }}=S_{f n i}$.

Equation (5) allows us to represent the probability $P\left(B_{f} \mid A_{i n}\right)$ of the transition of the system between the states characterized by the realization of events $A_{i n}$, and $B_{f}$ in the form

$$
\begin{aligned}
P\left(B_{f} \mid A_{\text {in }}\right)= & P\left(\bigcup_{n=1}^{N} \tilde{S}_{f n i}\right)=\sum_{n=1}^{N} P\left(S_{f n i}\right)+2 \sum_{n<m=1}^{N} g_{n m} P\left(S_{f n i} \cap S_{f m i}\right)+ \\
+ & 4 \sum_{n<m<k=1}^{N} g_{n m k} P\left(S_{f n i} \cap S_{f m i} \cap S_{f k i}\right)+\ldots+ \\
& +2^{N-1} g_{12 \ldots N} P\left(S_{f 1 i} \cap S_{f 2 i} \cap \ldots \cap S_{f N i}\right),
\end{aligned}
$$

Where $g_{n, m}, g_{n, m, k}, \ldots, g_{1,2, \ldots, N}=+1$ or -1 .

We represent equation (6) in a form that is more convenient for describing stochastic processes. It is possible to present the offered equation in a look

$$
\begin{gathered}
P\left(B_{f} \mid A_{i n}\right)=\sum_{n, m=1}^{N} g_{n m} P\left(S_{f n i} \cap S_{f m i}\right)+ \\
+4 \sum_{n<m<k=1}^{N} g_{n m k} P\left(S_{f n i} \cap S_{f m i} \cap S_{f k i}\right)+\ldots+2^{N-1} g_{12 \ldots N} P\left(S_{f 1 i} \cap S_{f 2 i} \cap \ldots \cap S_{f N i}\right),
\end{gathered}
$$

where $g_{n, m}=+1$ or $-1, n \neq m ; g_{n, n}=+1, n=m ; g_{n m k}=+1$ or $-1 ; \ldots$

To calculate the transition probabilities (7), or their analytical representation, it is necessary to specify the studied system.

\section{Physical interpretation of the equation for transitions in the space of random joint events}

Let's consider system which can stay in states $n=1,2 \ldots, N$. The system eventually makes transitions between states. The transition of the system from the state $n_{i n}$ at the time $t_{i n}$ to the state $n_{f}$ at the time $t_{f}>t_{i n}$ is carried out along a certain random trajectory determined by the set of numbers $n_{i n}, n_{1}, n_{2}, \ldots n_{f}$. Action of system is determined for each trajectory: $S\left[n_{i n}, n_{1}, n_{2}, \ldots, n_{N}, n_{f}\right]$. Action of system we shall present as: $S\left[n_{i n}, n, n_{f}\right]$, where $n\left(n_{1}, n_{2}, \ldots, n_{N}\right)$ a multiindex.

To events $B_{f}, A_{i n}$ we put in conformity of the states $n_{f}, n_{i n}$. To events $S_{f n i}$ we put in conformity the action of system $S\left[n_{i n} ; n ; n_{f}\right]$. The equation (7) in space of the states of system becomes

$$
\begin{gathered}
P\left(n_{f} \mid n_{i n}\right)=\sum_{n, m=1}^{N} g_{n m} P\left(S\left[n_{i n}, n, n_{f}\right], S\left[n_{i n}, m, n_{f}\right]\right)+ \\
+4 \sum_{n<m<k=1}^{N} g_{n m k} P\left(S\left[n_{i n}, n, n_{f}\right], S\left[n_{i n}, m, n_{f}\right], S\left[n_{i n}, k, n_{f}\right]\right)+\ldots+
\end{gathered}
$$




$$
\begin{gathered}
+2^{N-1} g_{12 \ldots N} P\left(S\left[n_{i n}, 1, n_{f}\right], S\left[n_{i n}, 2, n_{f}\right], \ldots, S\left[n_{i n}, N, n_{f}\right]\right) . \\
g_{n, m}=+1 \text { or }-1, n \neq m ; g_{n, n}=+1, n=m ; g_{n m k}=+1 \text { or }-1 ; . . g_{1,2, \ldots N}=+1 \text { or }-1 .
\end{gathered}
$$

Probabilities of transition in space of the states of system, where pairs joint event, becomes

$$
P\left(n_{f} \mid n_{i n}\right)=\sum_{n, m=1}^{N} g_{n m} P\left(S\left[n_{i n}, n, n_{f}\right], S\left[n_{i n}, m, n_{f}\right]\right)
$$

Where $g_{n, m}=+1$ or $-1, n \neq m ; g_{n, n}=+1, n=m$. We do a postulate

$$
\begin{gathered}
P\left(S\left[n_{i n}, n, n_{f}\right], S\left[n_{i n}, m, n_{f}\right]\right)=P_{n m}\left|\cos \left[S_{f n i}-S_{f m i}\right]\right|, \\
g_{n m}=\cos \left[S_{f n i}-S_{f m i}\right]\left|\cos \left[S_{f n i}-S_{f m i}\right]\right|^{-1} .
\end{gathered}
$$

Therefore

$$
P\left(n_{f} \mid n_{i n}\right)=\sum_{n, m=1}^{N} P_{n m} \cos \left[S_{f n i}-S_{f m i}\right] .
$$

The proposed physical model and equation (12) describe the diffraction of particles when passing through a diffraction grating with $\mathrm{N}$ gaps. In this model $n_{i n}$ means the emission state of a particle with momentum $\mathbf{p}, n_{f}$ - the state of the particle on the registration screen, which is parallel to the plane of the diffraction grating, $S[i n f]$ is the particle's action along the trajectory from state $n_{i} n$ to point $n_{f}$ passing through the slot $\mathrm{n}: S[i n f]=\mathbf{k r}_{\text {in }}+\mathbf{k r}_{n f}$, where $\mathbf{k}=(\mathbf{p}) \hbar^{-1}$ - particle wave number; $\mathbf{r}_{i n}, \mathbf{r}_{n f}$ - the vectors between points.

Probability (12) describes the distribution density of particles on the observation screen as the particle stream passes through the diffraction grating. This means that the particle trajectories are random pairwise joint events.

A quantum system with a discrete energy spectrum $E_{n}, n=1,2, \ldots$ was described by the method of functional integration in [18],[17]. The system makes transitions between quantum states $n=1,2, .$. under the action of a polarized monochromatic electromagnetic wave with frequency $\Omega$. The probability of a quantum transition $P\left(n_{f}, t_{f} \mid n_{i n}, t_{i n}\right)$ between the states $n_{i n}, n_{f}$ for the time interval $\left(t_{f}-t_{i n}\right)$ was obtained in the form:

$$
\begin{aligned}
& P\left(n_{f}, t_{f} \mid n_{i n}, t_{i n}\right)=\sum_{n_{K-1}=1, m_{K-1}=1}^{N} \ldots \sum_{n_{1}=1, m_{1}=1}^{N} \int_{0}^{1} \int_{0}^{1} \ldots \int_{0}^{1} \int_{0}^{1} d \xi_{0} d \zeta_{0} d \xi_{1} d \zeta_{1} \ldots d \xi_{N-1} d \zeta_{N-1} \times \\
& \times P_{f} \cos \left[S\left[n_{f} ; n_{K-1}, \xi_{K-1} ; \ldots n_{1}, \xi_{1} ; n_{i n}, \xi_{0}\right]-S\left[n_{f} ; m_{K-1}, \zeta_{K-1} ; \ldots m_{1}, \zeta_{1} ; n_{i n}, \zeta_{0}\right],\right.
\end{aligned}
$$

where the summation is carried out over all possible trajectories between the states $n_{i n}, n_{f}$. The action along any trajectory has the form

$$
\begin{aligned}
& S\left[n_{f} ; n_{R-1}, \xi_{K-1} ; \ldots n_{1}, \xi_{1} ; n_{i n}, \xi_{0}\right]=\sum_{k=1}^{K} S\left[n_{k}, n_{k-1}, \xi_{k-1}\right], \\
& \quad S\left[n_{k}, t_{k} ; n_{k-1}, t_{k-1} ; \xi_{k-1}\right]=2 \pi\left(n_{k}-n_{k-1}\right) \xi_{k-1}+\times \\
& \times \Omega_{n_{k} n_{k-1}}^{R}\left[\cos \left(2 \pi\left(n_{k}-n_{k-1}\right) \xi_{k-1}+\left(\Omega+\omega_{n_{k}, n_{k-1}}\right) \frac{t_{k}+t_{k-1}}{2}\right)+\right. \\
& \left.+\cos \left(2 \pi\left(n_{k}-n_{k-1}\right) \xi_{k-1}-\left(\Omega-\omega_{n_{k}, n_{k-1}}\right) \frac{t_{k}+t_{k-1}}{2}\right)\right]\left(t_{k}-t_{k-1}\right),
\end{aligned}
$$

where $n_{0}=n_{i n}, n_{N}=n_{f}, t_{0}=t_{i n}, t_{N}=t_{f} ; \omega_{n_{k}, n_{k-1}}$ - frequency of quantum transition of the system between states $n_{k}, n_{k-1} ; \Omega$ - frequency of electromagnetic field; $\Omega_{n_{k} n_{k-1}}^{R}$ - Rabi frequency for state $n_{k}, n_{k-1}[19]$. 
Formula (13) explains quantum processes in agreement with experiment.

An expression for the probabilities of state of a quantum system, analogous to (12), was proposed in [19],[20] in the method of functional integration along trajectories in the coordinate representation.

The coincidence of the expressions (12), (13) for the probabilities of quantum transitions makes it possible to assert that the proposed model of stochastic processes in the space of pairwise quantum joint events describes quantum processes.

\section{Conclusion}

The proposed model of joint quantum random events and equations written in the space of these events allows us to describe processes in quantum mechanics, provided that the events are only pairwise compatible.

It is of interest to study quantum processes in which there is a compatibility of random events of a higher order in accordance with the proposed equations.

\section{References}

[1] G.Greenstein, A.Zajonc, The Quantum Challenge Modern Research the Foundations of Quantum Mechanics. Jones and Bartlett Pulishers, lnc. 2006, page 296.

[2] V.Demutskii, R.Polovin, Sov. Phys. Usp. 35 (10), (10),page 857-896, (1992).

[3] G.Skorobogatov, S. Svertilov, Physical Review A, 58 , Number 5, page 3426-3432, (1998).

[4] N.Popov, Elements of the theory of quantum probabilities , (Computing Center RAS, Moscow), 1996, page 206.

[5] A.Holevo, Statistical structure of quantum theory, (Berlin, Heidelberg, New York, London, Paris, Tokyo, Hong Kong, Barselona, Budapest: Springer - Verlag, 2001) page 150.

[6] A.Holevo, Probabilistic and Statistical aspects of quantum theory, (Space Research Institute, Moscow,2003), page 410.

[7] A.Hrennikov, Non-Kolmogorov probability theory and quantum physics ,( Fizmatlit, , Moscow, 2003), page 208.

[8] A.Biryukov, Theoretical and Mathematical Physics. Samara, 7, page 56 -60, (1971).

[9] A.Biryukov, Theoretical physics. Samara, 10, page 22-31, (2009).

[10] A.Biryukov, Bulletin of Samara State University, naturally a scientific series, 7 , (78), page 137 - 144, (2010).

[11] A.Biryukov, Proceedings of science Qfthep 2010, 085, http://pos.sissa.it.

[12] A.Kolmogorov, Basic concepts of probability theory, (Science, Moscow, 1974,) page 120.

[13] Yu. Rozanov, Lectures on probability theory, (Science, Moscow, 1968,) page 120.

[14] A. Shiryaev, Probability, (Science, Moscow, 1989), page 640.

[15] N. Vereshchagin, A. Shen, The beginnings of set theory, (ICMMO, Moscow, 2008), page 128.

[16] A. Biryukov, M. Shleenkov, Theoretical physics. Samara, 13, page 8 - 42, (2012).

[17] A.Biryukov, Y.Degtyareva, M. Shleenkov, Bulletin of the Russian Academy of Sciences: Physics 2018, 82, Issue 12, page 1565-1569, (2018).

[18] M.Scully, M.Zubairy, , Quantum optics, (Cambridge University Press, 1997), page 512.

[19] G. Ryazanov, JETP, 35, 1, page 123-131, (1958).

[20] Yu.Kuharenko, 50 years of quantum mechanics , (Science, Moscow, 1979), page 126130. 